\title{
Dynamic scheduling of elevators by the Elevator Group Control System using a hybrid Approach
}

\author{
Malan D. Sale ${ }^{1}$, Dr. V. Chandra Prakash ${ }^{2}$ \\ ${ }^{1}$ Research Scholar, Department of Computer Science \& Engineering, KLEF, Vaddeswaram, Andhra Pradesh, \\ India,mdsale2006@gmail.com \\ ${ }^{2}$ Professor, Department of Computer Science \& Engineering, KLEF, Vaddeswaram, Andhra Pradesh, \\ India,vchandrap@kluniversity.in
}

\begin{abstract}
Elevator Group Control System (EGCS) has a vital role in the vertical transport operations in tall buildings. In this research work, a hybrid approach for the dynamic-scheduling of elevators(lifts) in EGCS to handle the down-peak and up-peak traffic conditions is proposed. The principal motive of this research is to minimize the factors viz. the passengers traveling time, average waiting time, and the passenger's inconvenience. The administrator of EGCS can set timeslots (which may be overlapped) both day-wise and date-wise in the elevator-control-information, which will be used by the EGCS The EGCS takes into account the administrator's elevator-control-information regarding various timeslots, both day-wise and date-wise, and estimates the amount of traffic timewise. The administrator can also indicate which elevators are in-service and out of service for proactive and reactive risk management. The anticipated hybrid approach can tackle several scenarios or problems while minimizing the factors mentioned above.
\end{abstract}

Key words: EGCS, Scheduling, Preferred Floors, Time-based Scheduling, Traffic-based Scheduling, Down-peak Traffic, Up-peak Traffic, Risk management in EGCS.

\section{INTRODUCTION}

To provide efficient transportation to today's increasing population is a very challenging issue. In high-rise buildings, the usage of elevators, in addition to stairways, improves the traveling satisfaction in vertical transportation. Using elevators to travel from the floor $\mathrm{i}$ to $\mathrm{j}$ reduces human effort and saves time. The people prefer an elevator to reach higher floors instead of using the stairs. For almost all officers, entrepreneurs, and working employees, "Time is money," this statement adds extra meaning and value.
Waiting for the elevator/lift may be only a moment, but it can be frustrating for many busy passengers. People face many problems in the traditional elevator system with only one elevator, like a more waiting queue for the elevator/lift and long ride time. With multiple elevators, the problem of elevator dispatching and coordination originates. EGCS is the solution. This research concentrates on the dynamic dispatching of lifts in the EGCS to handle the heavy-traffic situations in a commercial type building.

\subsection{Even-Odd scheduling}

The research proposed by Malan Sale and Dr. V. ChandraPrakash [1] introduces centralized even-odd scheduling in the EGCS. The even-odd elevator scheduling classifies the building stories into two sectors: odd stories and even stories. even floor calls are serviced by even-numbered elevators, and odd floor requests are fulfilled by odd-numbered elevators/lifts.

\subsection{Traffic based scheduling}

The research of Malan Sale and Dr. V. ChandraPrakash [2] focuses on scheduling in a commercial building with schools, offices, and shopping malls available on different floors. Heavy traffic floors are recognized by identifying requests coming from several floors. Heavy traffic floors are assigned as preferred floors in the traffic-based floor priority scheduling. Maximum elevators are assigned to heavy traffic floors, and fewer elevators are assigned to fewer traffic floors. The traffic-based scheduling is useful in the commercial building with variable traffic on specific floors throughout the day. The even-odd scheduling is not efficient in these buildings as heavy traffic is on specific floors of the building. The proposed research identifies the heavy traffic floors from the historical data and does the elevator allocation. More elevators are allotted to peak traffic floors, and fewer elevators are allotted to less peak traffic floors. Traffic-based scheduling is best suitable when different floors of the building have uneven traffic. 
Malan D. Sale et al., International Journal of Emerging Trends in Engineering Research, 8(9), September 2020, $6291-6297$

\subsection{Time-based scheduling}

The research proposed by Malan Sale and Dr. V. ChandraPrakash [3] focuses on time-based floor priority elevator scheduling in the EGCS. The research suggests the usage of elevators at a specific period to service the high priority floor requests. According to peak traffic timings, the priority is given to the floors, and elevators are assigned as per the scheduled times dynamically. In a commercial building, schools and offices are situated at various levels. In the morning, the upgoing traffic is high as everyone wishes to reach office \& school on time and arrives at the entrance floor; this type of traffic is called up-peak traffic. To handle the upward-peak traffic, elevators are allotted to serve the request from the entrance floor to preferred floors (school or office level) only.

Similarly, in the evening time, the down-going traffic is high as all want to reach the main entry floor from their respective floors; this type of traffic is called down-peak traffic. To handle the down-peak traffic, elevators are assigned to attend the request from the preferred floor to the entrance floor only. Some elevators are reserved for servicing the intermediate calls. The elevator scheduling is regular once the peak period is over.

Consider a scenario where school timing is 9.00 am to 4.00 $\mathrm{pm}$, and the office slot is $10.00 \mathrm{am}$ to $5.00 \mathrm{pm}$. In the morning between $9.00 \mathrm{am}-9.15 \mathrm{am}$, and $10.00 \mathrm{am}-10.15 \mathrm{am}$, there is up-peak traffic. Similarly, at 4.00 pm-4.15 and 5.00 pm-5.15 $\mathrm{pm}$, there is down-peak traffic. To handle such type of heavy traffic at a particular time, the time-based floor priority is the best suitable approach. Consider a scenario where the office and school timings are the same, and elevators are allotted to the office floor, it increases the inconvenience to the passengers who wish to reach the school floor, as elevators are not available to them. To handle such types of situations, the proposed system suggests the hybrid approach of elevator scheduling. The proposed hybrid system is the integration of even-odd, traffic-based, time-based, and risk management EGCS. In this hybrid approach, the controller identifies heavy traffic floors dependent on the population of the floor and assigns required elevators to floors. As elevators are assigned population-wise, the overlapped time problem is resolved.

\subsection{Risk management in EGCS}

If one of the elevators goes 'out of service' state, it causes inconvenience to the people using the elevator system. The building contains schools, hospitals, offices, shopping malls, etc. when any of the elevators fail may cause emergencies in the building. The hospital floor needs a continuous traveling facility to move or transfer patients or equipment from one ward to another. If the elevator assigned to the hospital floor fails, it may lead to dangerous conditions. To avoid such type of risk, the elevator rescheduling is required. So, if an allocated lift fails, a new elevator attends the request at the hospital floor. Once the elevator failure is detected, the projected system of Malan Sale and Dr. V. ChandraPrakash identifies the working elevators and reschedules the elevators as per the installed elevator configuration[4].

\section{LITERATURE SURVEY}

The study of Fujino, Atsuya, et al. [5] uses a floor-based attribute and car-based attribute control method. If the preferential level has a car call, the car with the least waiting time is preferred; for less crowding, the car with fewer passengers is chosen. The research of Covington and Michael A [6] uses MATLAB to build a simulation. The study focused on the defeasible logic with Prolog and d-prolog. In this logic, the rules have the form $\mathrm{A}$ : $\mathrm{B}$ : $\mathrm{C}$. If $\mathrm{A}$ is true and $\mathrm{B}$ is assumed true without contradiction, then conclude C. Fernandez, P. Cortes [7] gives the dynamic solution to the landing calls. The research uses a fuzzy logic algorithm to find the answer by considering the waiting time. The research study of Choongwan Koo et al.[8] focuses on zoning- based scheduling in the skyscraper construction building. In the skyscraper construction, an excessive quantity of workers and resources need to transport to their proper locations based on their roles. L Onieva, P. Cortes, J. Larraneta, [9] suggested that the elevators/lifts capacity is saturated in the upper floors in lunch-peak flow time \& departure time by neglecting the lower floor calls. All elevators are responding upper calls, so the waiting time of lower floors calls and intermediate calls is increased.

The study of C Ciflikli and E O Tartan [10] focuses on a parking algorithm for EGCS. Especially in pure incoming and up-peak traffic; this approach is equivalent to sending cars to the main entrance level. The study of Sungyong Ahna, et al. [11] concentrates on the dynamic variations in electricity charges and passenger traffic. The study suggests the use of more working elevators when the electricity charge is small, or the passenger traffic is more. The research of Ohhoon Kwon, et al. [12] proposes a Sensor Elevator Scheduling. They used floor sensors, RFID sensors, and video sensors to detect passenger's data prior to they impulse the calling button.

By using this data, the system controls the elevator operation and moving path. In the study of Suying Yang et al. [13] destir ation floor guidance method is used as a single input. The propose the dynamic floor partitioning to adjust the floor ectors based on traffic flow rate and passenger distribution. In the study of Lutfi Al-Sharif, Anas Riyal et al. [14], the destination control system (DCS) is anticipated to handle th uppeak traffic environments. The building is fragmented into several equal population sectors. Each sector is linked tp an elevator. When a call comes, the respective elevator frpm the sector is assigned. The research of Ronak Salamat, Bbrahimi, et al. [15] presents the routing algorithm for 3D-NoCs connected partially in a vertical way. When the 
Malan D. Sale et al., International Journal of Emerging Trends in Engineering Research, 8(9), September 2020, $6291-6297$

packet reaches a faulty elevator, the current node can pick a new elevator and reroute the packet toward a new elevator. The study of Seung-Tae park, bosuk yang [16], focusses on elevator fault analysis, risk management, and life-cycle assessment. The presented study analyzes the historical and survey data to identify the elevator failure so the maintenance team can use this information and recover the failure avoiding the risks.

The research proposed by Malan Sale and Dr. V. ChandraPrakash [17] focuses on the emergency elevator scheduling to handle the emergencies that occur in the building during fire breaks out, bomb threat detected, and the flood in the city. The survey in the field of EGCS is presented by Malan Sale and Dr. V. ChandraPrakash [18]. The research proposed by Malan Sale and Dr. V. ChandraPrakash [19] concentrates on the fuzzy-approach for the minimization of the waiting time.

The study of Hailiang Dong et al. [20] focuses on floor assignments to elevators based on fluctuant traffic. An overlapped range assignment algorithm selects an elevator to assist the series of floors along with the highest request floor. The focal goal is to transfer more passengers and not to decrease the figure of stops.
The research proposed by Jinghong Zheng [21] uses the dynamic time wrapping and clustering to find the traffic groups. Then according to this, data service zones are identified, and elevators are assigned to dispatch the passengers in each zone. The study [22] only discuses different scheduling techniques. They do not focus on centralized even-odd EGCS. The study [23] conducts an online survey of 468 people around 23 countries. The survey states that the majority of people are ready to opt elevators to vacate in emergencies. The study [24] concentrates on the Smoke spread system in a fire evacuation. Elevator halts on refuge level and ground level. The refuge level is one story below the affected fire floor.

\section{PROPOSED SYSTEM}

In the proposed system, the administrator (the responsible person looking elevator operations) can choose the required elevator configurations every day. He can set the heavy traffic floors and their preferred time slots weekday wise \& date wise, holiday list, and not working elevator information in the elevator-control-information (here it is a file). The data sets by the administrator, as shown in table 1, and accordingly, the EGCS switches the elevator scheduling.

Table 1: Day-wise and Date-wise timeslots for preferential floors

\begin{tabular}{|c|c|c|c|c|}
\hline $\begin{array}{l}\text { S. } \\
\text { No. }\end{array}$ & Day/Date & Timeslot floor i & Timeslot floor $\mathrm{j}$ & Timeslot floor $\mathrm{k}$ \\
\hline 1 & Monday & $09.00-9.15,17.00-17.15$ & $09.00-9.15,17.00-17.15$ & $10.00-10.15,18.00-18.15$ \\
\hline 2 & Tuesday & $19.00-19.15,12.00-12.15$ & $18.00-18.15,11.00-11.15$ & $17.00-17.15,09.00-9.15$ \\
\hline 3 & Wednesday & $18.00-18.15,10.00-10.15$ & $18.00-18.15,11.00-11.15$ & $19.00-19.15,12.00-12.15$ \\
\hline 4 & Thursday & $10.00-10.15,18.00-18.15$ & $10.00-10.15,18.00-18.15$ & $10.00-10.15,18.00-18.15$ \\
\hline 5 & Friday & $09.00-9.15,17.00-17.15$ & $10.00-10.15,18.00-18.15$ & $09.00-9.15,17.00-17.15$ \\
\hline 6 & Saturday & 0 & 0 & 0 \\
\hline 7 & Sunday & 0 & 0 & 0 \\
\hline 8 & $15-07-2020$ & $10.00-10.15,18.00-18.15$ & $10.00-10.15,18.00-18.15$ & $10.00-10.15,18.00-18.15$ \\
\hline 9 & $25-07-2020$ & $10.00-10.15,18.00-18.15$ & $11.00-11.15,18.00-18.15$ & $12.00-12.15,19.00-19.15$ \\
\hline 10 & $30-07-2020$ & $09.00-9.15,17.00-17.15$ & $09.00-9.15,17.00-17.15$ & $10.00-10.15,18.00-18.15$ \\
\hline
\end{tabular}


Malan D. Sale et al., International Journal of Emerging Trends in Engineering Research, 8(9), September 2020, $6291-6297$

The EGCS fetches the data from the elevator-control-information, and stores in the internal local storage (file pointer and an array), detects the system day and date, detects the call request time, and do the elevator scheduling. Table 1 . shows the sample of day-wise and date-wise timeslots set by the administrator in the elevator-control-information.

After every one-minute interval, the EGCS checks an external elevator-control-information to detect any changes made by the administrator. If any change in the input data store, EGCS updates the internal storage and reschedules the elevators accordingly. The administrator can choose the base scheduling algorithm to install as per the building requirements. For small buildings, the even-odd scheduling is best suitable. For commercial buildings with offices, shopping malls, and schools located on different floors, the traffic-based scheduling is useful, where elevator stops are decided depending on the flooring population. The EGCS accepts out-of-service elevator information from the elevator-control-information, finds the working elevators in the building, and do elevator scheduling as per the available elevators. The proposed EGCS has mainly four components: The data collection module, the recognition module, the scheduler, and the dispatcher.

The schematic of the projected hybrid approach in EGCS is described in Figure 1. The data collection module collects the data from the elevator-control-information; this data includes the day-wise and date-wise time slots to allocate to the preferred floors, holiday list, and working elevators from the administrator. As soon as the demand arises from the passenger, the recognition module recognizes the day, date, time, and assigned timeslot from the elevator-control-information. The scheduler checks the current day and date for a holiday. If the day and date are in the holiday list given in the elevator-control-information, the EGCS schedules the elevators as per the installed elevator scheduling (either even-odd scheduling or the traffic-based scheduling). If it is not a holiday, then the controller checks the day from the elevator-control-information and finds the timeslots.

If the given timeslots are overlapping slots and the call time is within the predefined time slot, the EGCS switches to a traffic-based time scheduling algorithm. In this approach, based on the floor population and available working elevators, the EGCS allocates elevators to preferred floors. If the given timeslots are not overlapping and the call time is within the predefined time slot, then EGCS switches to a time-based scheduling algorithm.

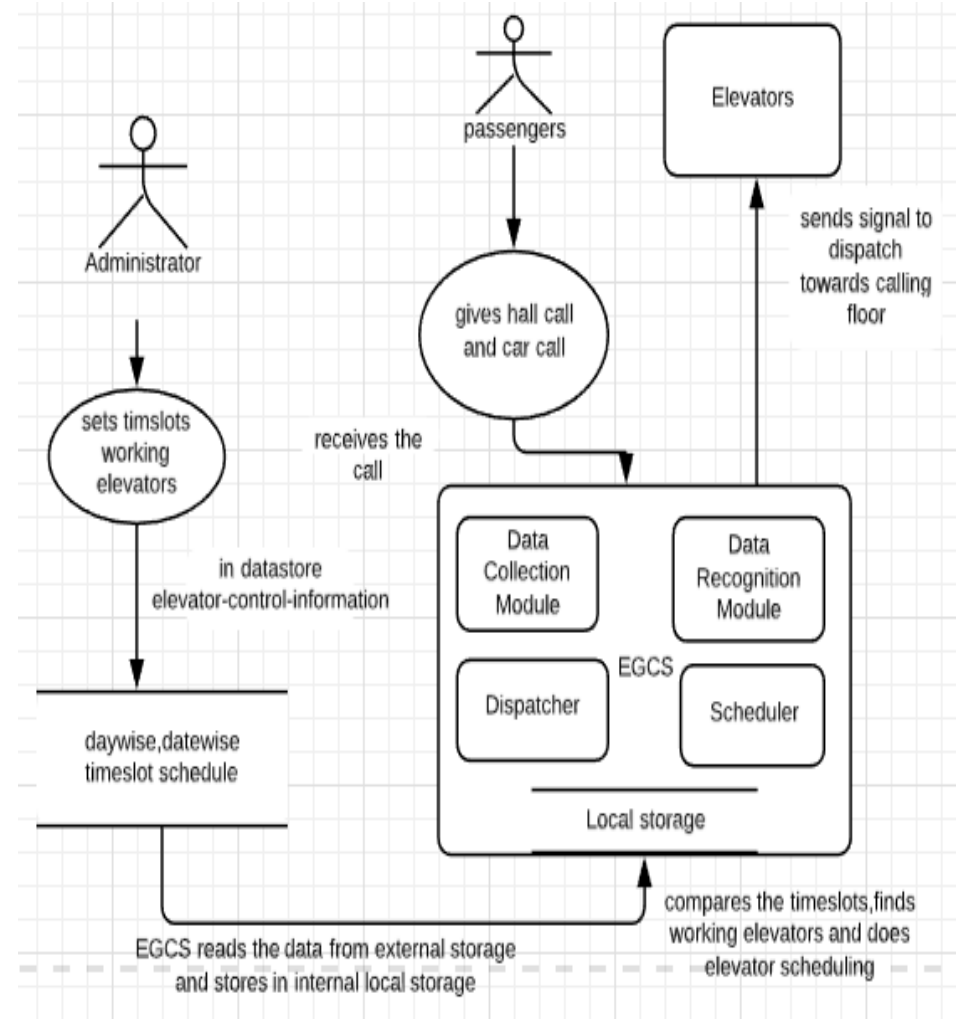

Figure 1: Proposed hybrid EGCS block diagram

In this approach, the EGCS checks the call time and given timeslots and accordingly allocate elevators to the proffered floor. If the call time is not within the predefined time slot, the EGCS switches to the installed base scheduling algorithm. The scheduler allocates the elevators as per the assigned timeslots and hybrid algorithm. Once the scheduling is done, the dispatcher sends a signal to assigned elevators to approach the requested floors and displays the list of elevator stops to the passengers. The passengers check the allocated elevators to their respective floors \& board into expected elevators.

The EGCS controller performs the following steps in the proposed hybrid algorithm:

1. recognize predefined high priority floors based on the intensity of requests, preferred time slots, and predefined holiday list, and working elevators.

a. To find working elevators

Count of Elevators $-\alpha$, Number of failed elevators $-\mu$, working elevators $-\beta$, even elevators $E$, Odd elevators $O$, Normal Elevators w

$$
\begin{aligned}
& \text { b. } \begin{array}{l}
\text { even-odd scheduling based on failed } \\
\text { elevators }
\end{array} \\
& \beta=(\alpha-\mu), E=\beta / 2, O=\beta / 2, w=\beta \% 2
\end{aligned}
$$


Malan D. Sale et al., International Journal of Emerging Trends in Engineering Research, 8(9), September 2020, $6291-6297$

2. get the current day and date

3. detect hall calls from the passengers

4. identify calling floor id

5. detect current day $\mathrm{D} \&$ date and compare with predefined holiday list $\mathrm{H}=\{\ldots$.

6. if holiday continue with installed base scheduling algorithm (even odd scheduling algorithm/traffic-based scheduling)

7. detect hall call request time $c_{t}$

8. if call time $\mathrm{C}_{t}$ is within the predetermined time slot $T_{t}$ and the day is not a holiday $\mathrm{D} \neq \mathrm{H}$, and preferred floors have the common time slot

9. switch to traffic-based time preference scheduling algorithm

a. count number of calls per level $c_{f}$

b. find call range for $\mathrm{p}=1 \ldots \ldots \mathrm{n}$ (e)

c. $\mathrm{s}_{\mathrm{p}}=(C \div \mathrm{n}(\theta)) \times \mathrm{p} \quad C$ constant factor-100

d. Lift allocation for each floor $\mathrm{f}_{\mathrm{i}}, \quad \mathrm{i}=1 \ldots n(f)$ for $\mathrm{p}=1 \ldots \ldots . \mathrm{n}(\mathrm{e})$

floor/Max Calls) $* 100$

if $\quad \mathrm{s}_{\mathrm{p}-1}<\mathrm{c}_{\mathrm{f}}<\mathrm{s}_{\mathrm{p}}$ then $\mathrm{c}_{\mathrm{f}}$-(calls per

e. $\quad k=n(e)-(n(e)-p) \quad$ where $s_{0}=0$;

f.assign $e_{0}, e_{1} \ldots \ldots e_{k}$ to respective floor $f_{i}$

g. if the incoming call is from $f_{i}$, check available elevators, and allocate to identified priority floors. Dispatch elevator to floor $\mathrm{f}_{\mathrm{i}}$ from the assigned elevator list based on the proposed assignment algorithms.

10. otherwise switch to the time-based scheduling algorithm

a. call time is within the given preferred floor time slot, then allocate elevators to floor

b. otherwise, continue with the installed base scheduling algorithm (even odd scheduling algorithm/traffic-based scheduling)

11. check if the timestamp expired

12. Once the timestamp will expire, switch to the base scheduling algorithm (even odd scheduling algorithm)

13. repeat the process whenever new data is available.

where,

$\mathrm{f}_{\mathrm{i}}$ : Building Floor, $\mathrm{n}$ (e): count of working elevators, e: Elevator, c: Number of request calls, $c_{\mathrm{f}}$ : Number of calls per floor, $\mathrm{k}$ : Number of elevators to allocate per, floor, $\mathrm{p}$ : Elevator allocation factor $\mathrm{p}$ lies in $1 \ldots \ldots \mathrm{n}(\mathrm{e}), \mathrm{s}_{\mathrm{p}}$ : Call Range.

\section{RESULTS}

The simulation is built for a building with forty-five floors and six elevators using JavaScript, jQuery. Each elevator has up and down buttons placed on each floor and destination floor buttons to give the destination input. Once the hall call is given, the controller passes a signal to the selected elevator, and the elevator dispatches to the requested floor. Consider a commercial building having preferential floors $\mathrm{i}, \mathrm{j}$, and $\mathrm{k}$, time to travel distance between two floors is $2 \mathrm{~s}$, door opening and closing time is $2 \mathrm{~s}$, and lift capacity is 10 passengers.

The waiting time is calculated by:

$$
\mathrm{T}=\left(\mathrm{d} * \mathrm{t}_{\mathrm{f}}\right)+\left(\mathrm{s} * \mathrm{t}_{\mathrm{d}}\right)
$$

Where, $\mathrm{T}$ - waiting time, d-total distance traveled in floors, $t_{f}$-time required to travel distance between two floors, $s$ number of elevator stops, $t_{d}$-time required to door open/close and halt on floor.

In the morning \& evening peak times, heavy calls will be for these floors and rare calls to remaining floors. In the existing dynamic zoning system, the building floors are split into various sectors as equal to the elevator count. When calls are coming, the system checks the elevator from the respective zone and sends it to the calling floor. If the elevator in the respective zone is full, the elevator from the upper zone is checked. If the upper zone elevator is full, lower-level elevators are checked. The elevator with the less crowd is sending to the calling floor. The system works well for an equal population in the sectors, but for the unequal floor population, it leads to long waiting times. As no express elevators to the heavy traffic floors and elevator from another zone may take additional stops to service the zone request, the waiting time of preferential floor calls is increased. In the proposed system, the express elevators are allotted to heavy traffic floors. Based on the traffic of the floor, the count of allotted elevators varies. For more traffic, more elevators, and for low traffic, fewer elevators are allotted. This minimizes the waiting time of preferential floor calls.

Table 2 and figure 2 gives the results obtained from the traditional sector-based arrangement and the presented hybrid system in the evening and morning peak time. As compared with sectoring scheduling, the time-based traffic floor preference reduces the elevator stops and waiting time \& journey time of passengers. 


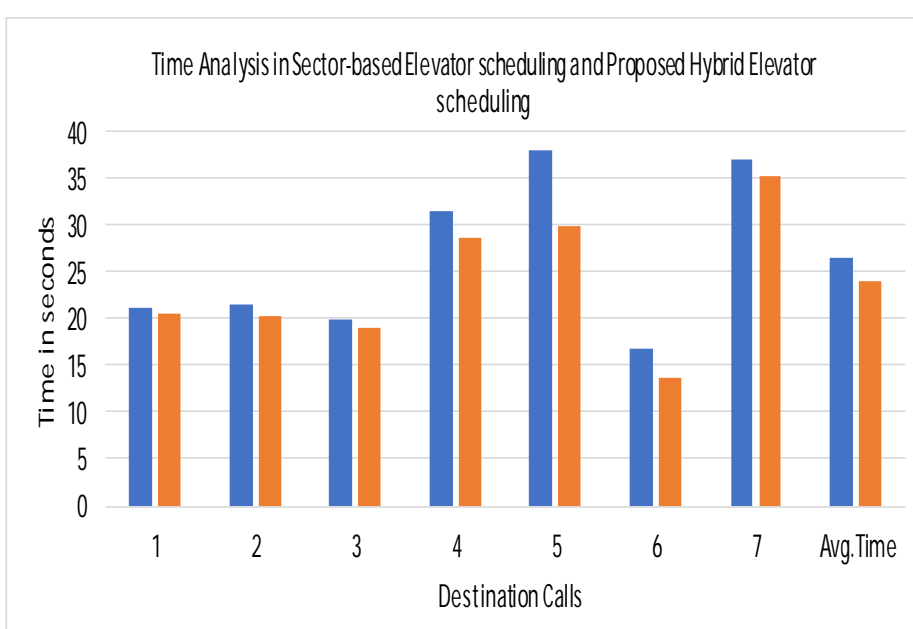

- Sector-Based Elevator Scheduling Hybrid Elevator Scheduling

Figure 2: Time analysis in sector-based scheduling and proposed hybrid elevator scheduling

\section{CONCLUSION}

This research shows that the dynamic approach with time-based traffic floor priority in even/odd systems and traffic-based systems improves an existing elevator system by minimizing the passenger's average waiting/traveling time. The primary mission of the group controller is to assign proper elevators as per the real-time floor demand and update their service zone. The successful implementation of a dynamic approach relies on a good monitoring system that evaluates the real-time traffic demand of every floor. The proposed hybrid approach gives a better solution to real-time traffic demands. The suggested hybrid approach with time-based and traffic-based floor priority is feasible and practical to reduce passengers waiting and journey time.

Future research can be carried by focusing on IoT based EGCS.

\section{REFERENCES}

1. Malan Sale, V. ChandraPrakash, "EGCS: scheduling of elevators using even and odd elevators approach," Advanced Research in Journal of dynamical and control-systems, issue 18, 3231-3242, 2017.

2. Malan Sale, V. ChandraPrakash, "Traffic-Based Floor Preference for the Scheduling of elevators in EGCS," Electrical Engg. Indonesian Journal of \& Computer Science, Vol. 16, No. 2, pp. 835-842 November 2019.

3. Malan Sale, V. Chandra Prakash, "Dynamic dispatching of elevators in EGCS with Time-based-floor-preference," Recent Tech. Int. Journal of \& Engineering, Vol-7 (5), January 2019.
4. Malan Sale, ChandraPrakash V., "EGCS with Riskmanagement," Int. Jour. of Engg. Research Emerging Trends in, 8(4), April pp. 1135 - 1141, 2020.

5. Atsuya, et al., "An EGCS with a floor-control method \& system-optimization using GA algorithms," Trans. IEEE on Industrial Elect., 44.4, 546-552, 1997.

6. Covington, Michael A., "Logical-control of an elev. with defeasible-logic," Trans. IEEE on Automatic Control, 45.7, pp. 1347-1349, 2000.

7. P. Cortes, et al., "Dynamic Fuzzy Logic EGCS with relative waiting-time consideration," Transactions IEEE on Industrial Electr., 61.9 pp. 4912-4919, 2014.

8. Jaeseong Yoon, Choongwan, Koo, "Zoning-Based vertical-transportation-optimization for-workers at peak-time in a skyscraper- construction," Civil infrastructure and Engg. Comp.-Aided 31 pp. 826-845, 2016.

9. P. Cortes, J. Larraneta, et al., "Genetic algorithm GA for controllers-in-elevator-groups: analysis \& simulation during lunch-peak-traffic," Applied jour. of soft-comp pp. 4159-174 2004.

10. C Ciflikli and E O Tartan, "Arrival Probability-Based Parking-algorithm for EGCS," Physics Journal of Conference Series 1425 012130, 2019.

11. Sungyong Ahna et at., "A smart-elevator-scheduler that considers dynamic-changes of energy-cost \& user-traffic," Computer-aided integrated Engg.,24, 2017.

12. Ohhoon Kwon, Hyokyung Bahn, Eunji Lee, "Sensor-aware elevators-scheduling for smart-building environments," Building \& Environment 72, 2014.

13. Suying Yang, et al., "Dynamic-partition of EGCS with destination-floor-guidance in the up-peak-traffic," Computers Journal of, 4(1), 2009.

14. Lutfi Al-Sharif et al., "Assessing the up-peak-performance of destination-EGCS using the real-time-allocation of landing calls," Int. J. Industrial \& Systems Engg., 25(4), 2017.

15. Ronak Salamat, et al., "A resilient-routing algo. with formal reliability-analysis for partially-connected3d-NOCs," transactions IEEE on computers, vol. 65 (11) November 2016.

16. Bo suk yang, Seung-Tae park, "Implementation of risk-based inspection for elevator maintenance," mechanical Journal of science and tech. 24 (12), 2010.

17. M. D. Sale, V. ChandraPrakash, "Elevators Scheduling in EGCS for the Emergency Evacuation of High-Rise Buildings," Journal of International Technology Research Scientific \&, volume 8, issue 12, Dec. 2019.

18. Malan Sale, V. Chandra Prakash, "Dynamic-dispatching of the elevator in EGCS: Research \& Survey," Int. Jou. of Innov. Tech \& Exploring Engg. ,8 (4), Feb. 2019.

19. Malan Sale, ChandraPrakash V., "Dynamic-scheduling of elevators with-reduced waiting-time of passengers 
Malan D. Sale et al., International Journal of Emerging Trends in Engineering Research, 8(9), September 2020, $6291-6297$

in EGCS: Fuzzy Approach," ICSE S. H., Springer, pp.339-346, 2017.

20. Hailiang Dong, et al., "Towards the Design of Optimal Range Assignment for Elevator Groups under Fluctuant Traffic Loads," IEEE 23rd ICERTCSA, Taiwan, 2017.

21. Jinghong Zheng, Tat Thomas Ho Chee, Yuan HuaiBing, "Traffic prediction of efficient elevator dispatching," Proceedings of TENCON IEEE, Korea, pp. 28-31, 2018.

22. Nagendra BD., "Optimization of Elevator Services Using Machine Learning," IRJEAT International Research Journal of Engg. \& Technology,03 (07) July 2016.

23. Kinsey, Michael \& Galea, E.R. \& Lawrence, Peter, "Human Factors Associated with the Selection of Lifts/Elevators or Stairs in Emergency and Normal Usage Conditions," Fire Technology, 48, 2012.

24. Cai, et al., "Numerical-studies on fire-hazards of elevator-evacuation in supertall-buildings," sage pub., Env. Indoor \& built, 2018. 